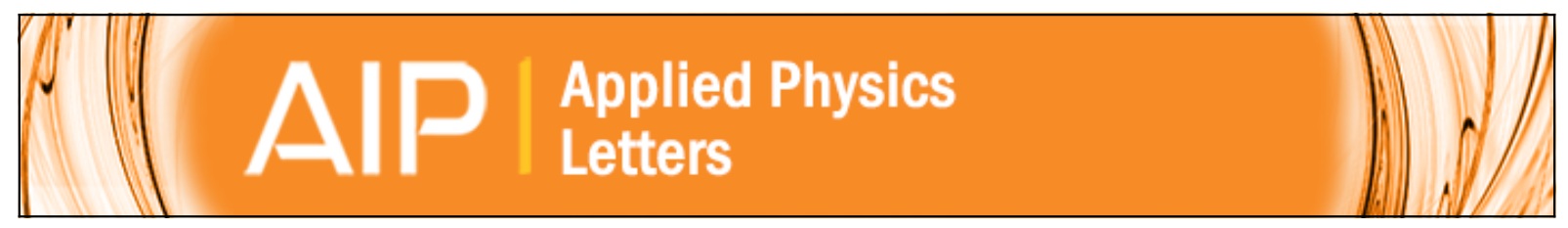

\title{
Tuning exchange bias
}

P. Miltényi, M. Gierlings, M. Bamming, U. May, G. Güntherodt, J. Nogués, M. Gruyters, C. Leighton, and Ivan K. Schuller

Citation: Applied Physics Letters 75, 2304 (1999); doi: 10.1063/1.124998

View online: http://dx.doi.org/10.1063/1.124998

View Table of Contents: http://scitation.aip.org/content/aip/journal/apl/75/15?ver=pdfcov

Published by the AIP Publishing

\section{Instruments for advanced science}
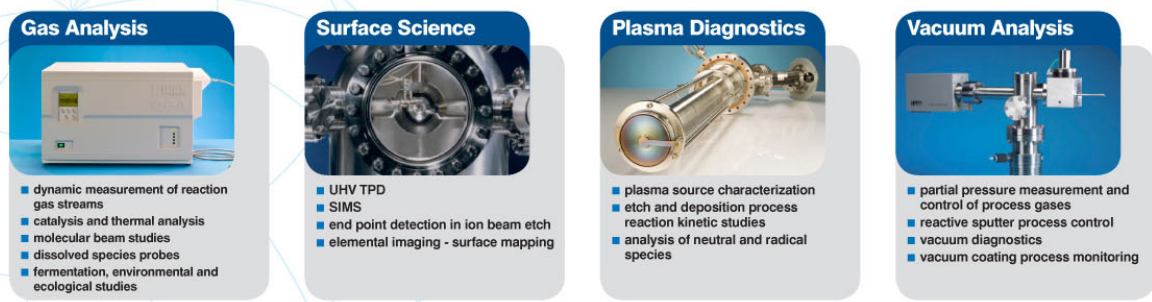

contact Hiden Analytical for further details

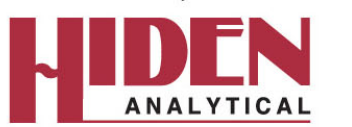

info@hideninc.com www.HidenAnalytical.com CLICK to view our product catalogue 


\section{Tuning exchange bias}

P. Miltényi, ${ }^{a)}$ M. Gierlings, M. Bamming, U. May, and G. Güntherodt

2. Physikalisches Institut der RWTH Aachen, 52056 Aachen, Germany

J. Nogués

Departament de Física, Universitat Autònoma de Barcelona, 08193 Bellaterra, Spain

M. Gruyters

Hahn-Meitner Institut Berlin, Glienicker Str. 100, 14109 Berlin, Germany

C. Leighton and Ivan K. Schuller

Physics Department 0319, University of California-San Diego, La Jolla, California 92093-0319

(Received 6 November 1998; accepted for publication 16 August 1999)

The exchange bias shift of the hysteresis loop, $H_{E}$, in antiferromagnetic/ferromagnetic layer systems can be easily controlled (within certain limits) by cooling in zero field from different magnetization states above the antiferromagnetic Néel temperature, $T_{N}$. This indicates that for moderate cooling fields, $H_{E}$ is determined by the magnetization state of the ferromagnet at $T_{N}$, and not by the strength of the cooling field. (C) 1999 American Institute of Physics.

[S0003-6951(99)01941-5]

Exchange bias is the shift of the ferromagnetic hysteresis loop along the field axis produced by an unidirectional exchange anisotropy at the interface between ferromagnetic (FM) and antiferromagnetic (AFM) films. ${ }^{1}$ Usually, to induce this unidirectional anisotropy and thus exchange bias, $\mathrm{AFM} / \mathrm{FM}$ layer systems are cooled (or grown) below the AFM Néel temperature, in the presence of a static magnetic field, generally a few times larger than the FM coercive field, $H_{C} \cdot{ }^{1-8}$ Larger cooling fields (i.e., well above the saturation field of the FM layer) do not further affect the FM layer. However, if the cooling field is large enough, it can affect the AFM layer. Thus, new phenomena such as a reduction or increase of $H_{E}$ at large cooling fields ${ }^{8-11}$ or "positive" exchange bias can be observed. ${ }^{8}$ If samples are cooled in zero field $\left(H_{\text {cool }}=0\right)$ from a demagnetized state the loops remain unshifted $\left(H_{E}=0\right){ }^{2,3,12-14}$ However, there is some evidence that shifted loops $\left(H_{E} \neq 0\right)$ can be obtained even when the samples are cooled in $H_{\text {cool }}=0$, if the cooling procedure starts from a remanent state. ${ }^{15-17}$ If the samples are cooled in $H_{\text {cool }}<H_{C}$ in the initial virgin magnetization curve, then the loop shift is much smaller than the one obtained from $H_{\text {cool }}$ $>H_{C} .^{3}$

In this letter we show that the exchange bias field, $H_{E}$, can be tuned by cooling in zero field from different magnetization states, indicating that the role of the cooling field is not to induce $H_{E}$ but only to have a single FM domain state above $T_{N}$ and thus a maximum exchange bias effect.

This is of obvious technological importance: It allows one to select the desired value of exchange bias via a simple postgrowth cooling procedure. For example, for the case of hybrid spin valve sensors the value of $H_{E}$ can be tuned such that the field induced resistance change occurs at very small applied fields.

We studied two different exchange bias systems, $\mathrm{FeF}_{2}$ $(\mathrm{AFM}) / \mathrm{Fe}(\mathrm{FM})$ and $\mathrm{CoO}(\mathrm{AFM}) / \mathrm{Co}(\mathrm{FM})$, both of which

${ }^{a)}$ Electronic mail: peter.miltenyi @physik.rwth-aachen.de allow for easy cooling and warming the samples across the AFM Néel temperature, $T_{N}\left(\mathrm{FeF}_{2}\right)=78 \mathrm{~K}$ and $T_{N}(\mathrm{CoO})$ $=291 \mathrm{~K}$.

The $\mathrm{FeF}_{2} / \mathrm{Fe}$ samples were prepared using electron beam deposition. $90 \mathrm{~nm}$ of $\mathrm{FeF}_{2}$ were deposited at a rate of 0.2 $\mathrm{nm} / \mathrm{s}$ at a substrate temperature $T_{S}=200^{\circ} \mathrm{C}$ on $\mathrm{MgO}$ (100) substrates, followed by a $12 \mathrm{~nm} F e$ layer, at a rate of 0.1 $\mathrm{nm} / \mathrm{s}$, at $T_{S}=150^{\circ} \mathrm{C}$ and a $3 \mathrm{~nm}$ capping layer of $\mathrm{Al}$, at a rate $0.1 \mathrm{~nm} / \mathrm{s}$, at $T_{S}=150{ }^{\circ} \mathrm{C}$. The base pressure of the chamber was better than $1 \times 10^{-7}$ mbar and the pressure during deposition was lower than $3 \times 10^{-6}$ mbar. The thickness of the different layers was controlled by a calibrated quartz oscillator. The $\mathrm{FeF}_{2}$ layer grows in the (110) orientation. ${ }^{18}$

The $\mathrm{Co} / \mathrm{CoO}$ samples were grown in a molecular beam epitaxy chamber with a base pressure of $5 \times 10^{-11}$ mbar. A Co layer of $8 \mathrm{~nm}$ was grown at room temperature at a rate of $0.3 \mathrm{~nm} / \mathrm{min}$ on a hydrogen terminated $\mathrm{Si}(111)$ substrate. ${ }^{19,20}$ The CoO layer $(100 \mathrm{~nm})$ was deposited at a rate of $1 \mathrm{~nm} / \mathrm{min}$ by evaporating $\mathrm{Co}$ in an oxygen atmosphere of 2 $\times 10^{-6}$ mbar using a substrate temperature of $T_{S}=110^{\circ} \mathrm{C}$. The chemical composition of the $\mathrm{CoO}$ layer was controlled with quantitative Auger spectroscopy. Electron diffraction [reflection high-energy electron diffraction (RHEED), lowenergy electron diffraction (LEED)] indicate a (111) orientation of the $\mathrm{CoO}$ layer.

For the $\mathrm{FeF}_{2} / \mathrm{Fe}$ samples the hysteresis loops were measured at $T=10 \mathrm{~K}$ using a superconducting quantum interference device (SQUID) magnetometer, applying fields up to \pm 2000 Oe. If the sample was cooled through $T_{N}$, as is customary, in a field large enough to saturate the FM layer, $H_{\text {cool }}=+2000$ Oe, but small enough not to affect the AFM layer, the loop shift at $T=10 \mathrm{~K}$ was $H_{E}=-330 \mathrm{Oe}$. We denote this field by $H_{E \text {, } \max }$. For the remaining discussion, the samples were cooled through $T_{N}$ in zero field $\left(H_{\text {cool }}=0\right)$. However, different magnetization states, $m(H=0)$, were set up at $T=85 \mathrm{~K}$, i.e., $T>T_{N}$, before the zero field cooling procedure. To obtain the different magnetization states minor 


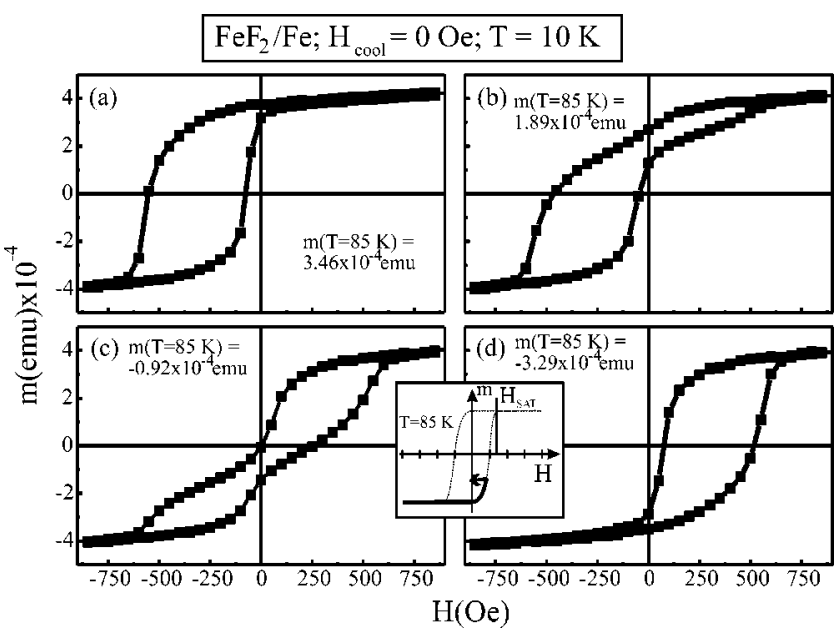

FIG. 1. Hysteresis loops for a $\mathrm{FeF}_{2} / \mathrm{Fe}$ bilayer at $T=10 \mathrm{~K}$ cooled in zero field $\left(H_{\text {cool }}=0\right)$ from $T=85 \mathrm{~K}$ in different magnetization states, (a) $m$ $=+3.46 \times 10^{-4}$, (b) $m=+1.89 \times 10^{-4}$, (c) $m=-0.92 \times 10^{-4}$, and (d) $m$ $=-3.29 \times 10^{-4} \mathrm{emu}$. Note that $m_{R}=3.54 \times 10^{-4} \mathrm{emu}$. The inset shows the procedure to set up different magnetization states at $T=85 \mathrm{~K}$. The lines are guides to the eye.

loops were carried out. As shown in the inset of Fig. 1, the sample was saturated in a negative field of, e.g., $H$ $=-4 H_{C}$. Then the field was increased to $0<H<+H_{\mathrm{SAT}}$, where $H_{\mathrm{SAT}}$ is the saturation field (see inset Fig. 1) and finally the field was reduced back to $H=0$. This procedure establishes different magnetizations above $T_{N}$ in the range $\pm m_{R}$, where $m_{R}$ is the remanent moment of the FM.

The magnetization was recorded during the cooling procedure, showing that the magnetization did not change more than $1.5 \%$ between $T=85 \mathrm{~K}$ and $T_{N}=78 \mathrm{~K}$ and not more than $4 \%$ between $T=85$ and $10 \mathrm{~K}$. If the sample is cooled in $H_{\text {cool }}=0$ from remanence $m_{R}$, the loop shift is similar to the one for field-cooled samples, ${ }^{1}$ i.e., $H_{E} \approx-\left|H_{E \text {, } \max }\right|$ (see Figs. 1 and 2). Nevertheless, if the sample is cooled in $H_{\text {cool }}=0$ from negative remanence, i.e., $m(H=0)=-m_{R}$, the loop shifts in the opposite direction, i.e., $H_{E} \approx+\left|H_{E \text {, } \max }\right|$ (similar to what is observed in samples cooled in negative fields ${ }^{18}$ ). These observations are in agreement with previous studies in other systems. ${ }^{15-17}$ For intermediate magnetizations $\mid m(H$ $=0) \mid<m_{R}$, the loops shift continuously between $+\left|H_{E, \max }\right|$ and $-\left|H_{E \text {,max }}\right|$ (Figs. 1 and 2). Thus, we can tune $H_{E}$ by

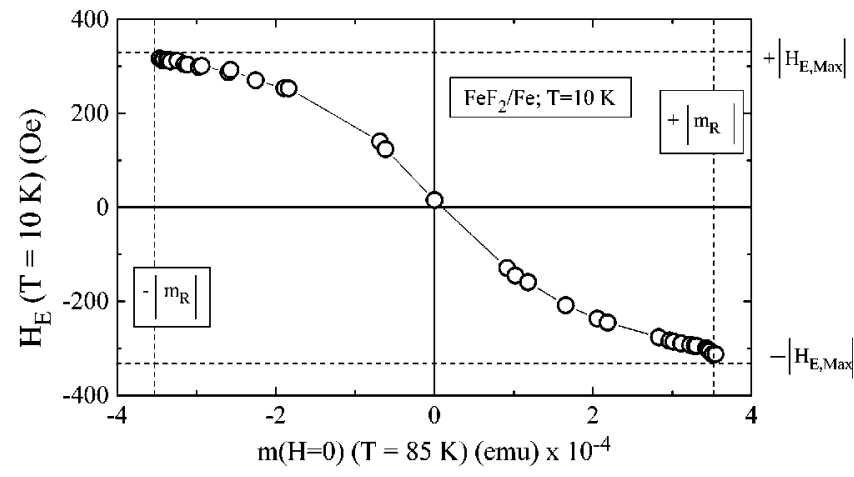

FIG. 2. Dependence of the exchange bias field, $H_{E}$, of a $\mathrm{FeF}_{2} / \mathrm{Fe}$ bilayer at $T=10 \mathrm{~K}$, on the zero field magnetic moment, $m(H=0)$, at $T=85 \mathrm{~K}$ before the zero field cooling procedure. The solid line through the data points is a guide to the eye. The dashed lines indicate the maximum attainable exchange bias fields at $T=10 \mathrm{~K},+\left.H_{E \text { inas }}\right|_{2}$ and the remanent moments at $T$ $=85 \mathrm{~K}, \pm\left|m_{R}\right|$.

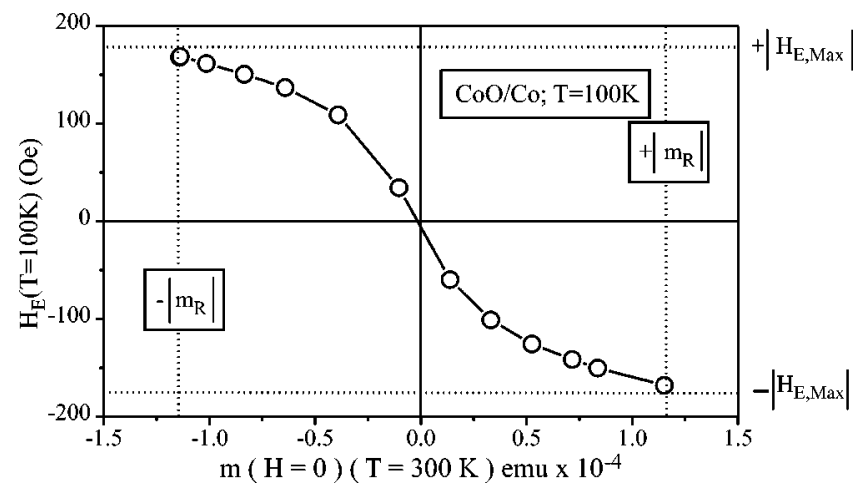

FIG. 3. Dependence of the exchange bias field, $H_{E}$, of $\mathrm{CoO} / \mathrm{Co}$ at $T$ $=100 \mathrm{~K}$ on the zero field magnetic moment, $m(H=0)$, at $T=300 \mathrm{~K}$ before the zero field cooling procedure. The solid line through the data points is a guide to the eye.

setting up the appropriate magnetization state, $m(H=0)$, above the Néel temperature before the zero-field cooling procedure.

The same experimental procedure was carried out with a $\mathrm{CoO} / \mathrm{Co}$ sample using a temperature of $T=300 \mathrm{~K}>T_{N}$ $=291 \mathrm{~K}$ to perform the minor loops in order to set up the different remanent magnetization states. The hysteresis loops were measured at a temperature of $100 \mathrm{~K}$ applying fields up to \pm 1000 Oe. The results shown in Fig. 3 exhibit an almost identical dependence of the exchange bias field on the initial magnetization as found above for the $\mathrm{FeF}_{2} / \mathrm{Fe}$ system.

These results indicate that the shift $H_{E}$ is determined by the magnetization state at $T=T_{N}$ and not by $H_{\text {cool }}$ (for moderate cooling fields), i.e., exchange bias can be obtained and $H_{E}$ be controlled without a cooling field.

These results are of particular interest for device fabrication where exchange bias is employed for pinning the magnetization direction of a ferromagnetic layer (e.g., spin valve sensors ${ }^{21}$ magnetoresistance devices $\left.{ }^{22}\right)$. $H_{E}$ usually is obtained from cooling or film deposition in an external field and $H_{E}$ is determined by the FM and AFM layer thicknesses and their microstructures. ${ }^{23,24}$ Here we demonstrated that $H_{E}$ can be tuned after device fabrication.

As shown in Fig. 1, the hysteresis loops of the $\mathrm{FeF}_{2} / \mathrm{Fe}$ samples consist of two components, i.e., two loops shifted to positive or negative fields with different relative weights depending on the magnetization state before cooling. The same behavior is found for the $\mathrm{CoO} / \mathrm{Co}$ samples (not shown). For the cases with $m<m_{R}$ which exhibit two components of the hysteresis loops, we infer that the FM layer for $T>T_{N}$ is divided into domains with orientations parallel and antiparallel to the field direction during the minor loop. During cooling each ferromagnetic domain determines locally the exchange bias in the area of the domain. At low temperatures, these areas with different exchange bias shift the loop in opposite directions. Thus, it is the relative amount of one or the other type of domain which controls the shift of the loop. This is confirmed by the behavior of the hysteresis loop when the system is cooled starting from $m(H=0)=0$ (see Fig. 4). If the sample is cooled in $m(H=0)=0$ as obtained from a minor loop procedure, an unshifted loop with two symmetrical components, one shifted to positive fields and one shifted to negative fields, is obtained (Fig. 4). However, if the sample is cooled in $m(H=0)=0$ as obtained from a 


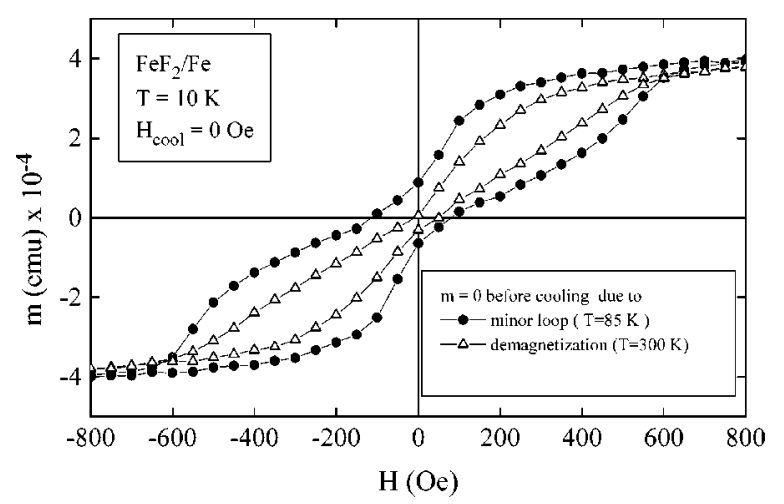

FIG. 4. Hysteresis loops for a $\mathrm{FeF}_{2} / \mathrm{Fe}$ bilayer at $T=10 \mathrm{~K}$ obtained by zero field cooling from $T=85 \mathrm{~K}$ in $m(H=0)=0$ set up by a minor loop procedure (full circles) and a high temperature $(T=300 \mathrm{~K})$ demagnetization procedure (open triangles). The solid lines through the data points are guides to the eye.

demagnetization procedure (at $T=300 \mathrm{~K}$ ), the two component feature of the loop is much less pronounced (Fig. 4). The demagnetization process reduces the size of the domains and randomizes their directions, thus the effect of the domains is greatly reduced. These results indicate that the role of $H_{\text {cool }}$ is only to ensure that the FM layer is in a uniformly magnetized state, which will guarantee the homogeneity (i.e., only one component) of the shifted loops at low temperatures. It is noteworthy that these results also confirm that magnetometry measures the average coupling over the whole interface area.

In conclusion, we have shown that the exchange bias field $H_{E}$ can be tuned between $\pm\left|H_{E \text {, max }}\right|$ by cooling in zero field from different magnetization states set up by minor loops slightly above $T_{N}$. This indicates that it is the magnetic state of the FM layer at $T_{N}$ and not the cooling field which controls the exchange bias below $T_{N}$. This gives the opportunity to tune the exchange bias even after device fabrication.

Note added in proof: After submission of this letter it came to our attention that similar work on CoO/Permalloy has been carried out by Gökemejier and Chien, J. Appl. Phys. 85, 5516 (1999).
This work was supported by the Deutsche Forschungsgemeinschaft through SFB 341 and by the U.S. Dept. of Energy. J. N. thanks the Spanish Ministerio de Educación y Cultura for its financial support.

${ }^{1}$ For a recent review, see J. Nogués and Ivan K. Schuller, J. Magn. Magn. Mater. 192, 203 (1999).

${ }^{2}$ W. H. Meiklejohn and C. P. Bean, Phys. Rev. B 102, 1413 (1956).

${ }^{3}$ M. Takahashi, A. Yanai, S. Taguchi, and T. Suzuki, Jpn. J. Appl. Phys. 19, 1093 (1980).

${ }^{4}$ R. Jungblut, R. Coehoorn, M. T. Johnson, J. ann de Stegge, and A. Reinders, J. Appl. Phys. 75, 6659 (1994).

${ }^{5}$ S. Soeya, H. Hoshiya, R. Arai, and M. Fuyama, J. Appl. Phys. 81, 6488 (1997).

${ }^{6}$ M. Tsunoda, Y. Tsuchiya, M. Konoto, and M. Takahashi, J. Magn. Magn. Mater. 171, 29 (1997).

${ }^{7}$ T. Lin, C. Tsang, R. E. Fontana, and J. K. Howard, IEEE Trans. Magn. 31, 2585 (1995).

${ }^{8}$ J. Nogués, D. Lederman, T. J. Moran, and Ivan K. Schuller, Phys. Rev. Lett. 76, 4624 (1996); J. Nogués, T. J. Moran, D. Lederman, Ivan K. Schuller, and K. V. Rao, Phys. Rev. B 59, 6984 (1999).

${ }^{9}$ T. J. Moran and Ivan K. Schuller, J. Appl. Phys. 79, 5109 (1996).

${ }^{10}$ T. Ambrose and C. L. Chien, J. Appl. Phys. 83, 7222 (1998).

${ }^{11}$ J. Nogués, D. Lederman, B. A. Gurney, and Ivan K. Schuller (unpublished).

${ }^{12}$ Y. Tsuchiya, K. Kosuge, S. Yamaguchi, and N. Nakayama, Mater. Trans., JIM 38, 91 (1997).

${ }^{13}$ X. Lin, G. C. Hadjipanayis, and S. I. Shah, J. Appl. Phys. 75, 6676 (1994).

${ }^{14}$ M. B. Stearns, J. Appl. Phys. 55, 1729 (1984).

${ }^{15}$ K. O’Grady, S. J. Greaves, and S. M. Thompson, J. Magn. Magn. Mater. 156, 253 (1996).

${ }^{16}$ H. Danan, H. Gengnagel, J. Steinert, and A. Linzen, J. Phys. Colloq. 32, C1-131 (1971).

${ }^{17}$ G. Bottoni, D. Candolfo, and A. Cecchetti, J. Magn. Magn. Mater. 155, 297 (1996).

${ }^{18}$ J. Nogués, D. Lederman, T. J. Moran, Ivan K. Schuller, and K. V. Rao, Appl. Phys. Lett. 68, 3186 (1996).

${ }^{19}$ H. E. Hessel, A. Feltz, M. Reiter, U. Memmert, and R. J. Behm, Chem. Phys. Lett. 186, 275 (1991).

${ }^{20}$ G-J. Pietsch, U. Köhler, and M. Henzler, J. Appl. Phys. 73, 4797 (1993).

${ }^{21}$ B. Dieny, V. S. Speriosu, S. S. P. Parkin, J. C. Scott, B. A. Gurney, D. R. Wilhoit, and D. Mauri, Phys. Rev. B 43, 1297 (1991).

${ }^{22}$ C. Tang, J. Appl. Phys. 55, 2226 (1984).

${ }^{23}$ A. P. Malozemoff, Phys. Rev. B 35, 3679 (1987).

${ }^{24}$ T. C. Schulthess and W. H. Butler, Phys. Rev. Lett. 81, 4516 (1998). 